\title{
Molecular Polymorphism and Phenotypic Diversity in the Eutypa Dieback Pathogen Eutypa lata
}

\author{
Renaud Travadon and Kendra Baumgartner
}

First author: Department of Plant Pathology, University of California, One Shields Avenue, Davis, CA 95616; and second author: United States Department of Agriculture-Agricultural Research Service, Crops Pathology and Genetics Research Unit, Davis, CA 95616. Accepted for publication 22 July 2014

\section{ABSTRACT}

Travadon, R., and Baumgartner, K. 2015. Molecular polymorphism and phenotypic diversity in the Eutypa dieback pathogen Eutypa lata. Phytopathology 105:255-264.

Pathogen adaptation to different hosts can lead to specialization and, when coupled with reproductive isolation, genome-wide differentiation and ecological speciation. We tested the hypothesis of host specialization among California populations of Eutypa lata (causal fungus of Eutypa dieback of grapevine and apricot), which is reported from $>90$ species. Genetic analyses of nine microsatellite loci in 182 isolates from three hosts (grapevine, apricot, and willow) at three locations were complemented by cross-inoculations on cultivated hosts grapevine and apricot to reveal patterns of host specialization. The cultivated hosts are likely more important sources of inoculum than the wild host willow, based on our findings of higher pathogen prevalence and allelic richness in grapevine and apricot. High levels of gene flow among all three hosts and locations, and no grouping by clustering analyses, suggest neither host nor geographic differentiation. Cross-inoculations revealed diversified phenotypes harboring various performance levels in grapevine and apricot, with no apparent correlation with their host of origin. Such phenotypic diversity may enable this pathogen to persist and reproduce as a generalist. Regular genetic reshuffling through sexual recombination, frequent immigration among hosts, and the lack of habitat choice in this passively dispersed fungus may prevent fixation of alleles controlling host specialization.
Host-pathogen coevolution in agroecosystems provides unique opportunities for studying rapidly evolving plant-microbe interactions (43). In contrast to host plants in natural ecosystems, many cultivated crops are the result of long-term breeding programs; crop species are selected by humans and thus do not participate in a completely natural way in the evolutionary "arms race" with pathogens. Rather, they constitute dense and genetically uniform host populations facilitating pathogen transmission between infected and noninfected plants, thus promoting large pathogen population sizes where selection is efficient. Moreover, host defenses impose divergent selection pressures, and pathogens have higher population sizes, generation times, mutation rates, recombination rates, and dispersal abilities than their host plants (18). These characteristics theoretically favor host specialization in the pathogen population. The role of host specialization in sympatric speciation of fungal plant pathogens has been documented in the literature, and many species of pathogens thought to have broad host ranges have been shown instead to represent multiple, host-specialized populations $(16,20,22,34,42)$.

Host specialization can be qualitative, such that the pathogen is unable to infect plant species other than the principal host, or quantitative, such that the pathogen has weak performance (i.e., decreased aggressiveness or lower reproductive fitness) on uncommon hosts. In the case of quantitative host adaptation, different host species are expected to vary in their importance in disease transmission, and overall disease incidence can sometimes reflect the host community's composition (7,27). Accordingly, identifying the host species that contributes most to disease spread can help practitioners design effective disease-control strategies (17).

Corresponding author: K. Baumgartner; E-mail address: kbaumgartner@ucdavis.edu

http://dx.doi.org/10.1094/PHYTO-04-14-0117-R

This article is in the public domain and not copyrightable. It may be freely reprinted with customary crediting of the source. The American Phytopathological Society, 2015.
In agricultural landscapes, i.e., a mosaic of crops exerting differential selective pressures on pathogen populations, the distribution of genetic variation (i.e., population genetic structure) in pathogen populations often coincides with the spatial heterogeneity of hosts (20). Host heterogeneity impacts two evolutionary forces: selection and gene flow. Frequently, populations exploiting different resources are genetically differentiated. For fungal plant pathogens, this pattern usually results from the divergent selection pressures exerted by distinct host plants (20), whereas active habitat choice, which limits migration among environments, is far less relevant for passively dispersed organisms such as fungi.

The ascomycete fungus Eutypa lata (Pers:Fr.) Tul. \& C. Tul. (=Eutypa armeniacae Hansf. \& Carter) (Xylariales, Diatrypaceae) is considered a wide host range pathogen, based on the fact that it is reported to infect and sporulate on a worldwide total of 70 genera (37 families) of host plants (15). However, reports of hosts that suffer from Eutypa dieback (i.e., severe disease symptoms) are restricted to cultivated hosts, namely grapevine and apricot (8). E. lata is found in Europe, North America, Australia, and South Africa. Recent investigation of its worldwide population genetic structure suggests a European origin of the fungus, with its current global distribution likely resulting from multiple introductions of genetically diverse genotypes into new areas (North America, Australia, and South Africa) via transport of infected plant material (45).

During the dormant (rainy) season, wind-dispersed ascospores infect the woody stems of the host through pruning wounds (8), and establish a localized infection (wood canker) that kills distal shoots (dieback). The mycelium decays the wood, in part through production of cell-wall-degrading enzymes (41). The translocation of phytotoxic fungal metabolites, via the vascular system, from infected wood to the herbaceous part of the plant is believed to be responsible for the characteristic misshapen, dwarfed leaves $(28,30)$. Mating occurs between compatible mycelia within the infected wood, resulting in the formation of ascospores $(8,35)$. 
The relative importance of specific hosts in the spread of Eutypa dieback to healthy vineyards is unclear, in part based on conflicting evidence of host specialization. For example, Trouillas and Gubler (46) found no evidence of host specificity; isolates originating from different hosts were all able to infect grapevine and there was no apparent phylogenetic grouping according to either host or geographic origins. As such, all hosts might be thought to be sources of inoculum. In contrast, a previous study found that isolates from grapevine and pear were pathogenic on apricot and almond but not consistently on pear (9). These authors suggested that grapevine is susceptible to a wide range of isolates, of which only a few are also infectious on hosts other than grapevine (in this case, pear). In other words, the pathogenic ability to infect particular hosts may be a unique feature of particular isolates.

We evaluated the host specificity of E. lata in California, based on population genetics analyses of the pathogen from three hosts (grapevine, apricot, willow). We tested the hypothesis that pathogen populations from different hosts or locations are genetically differentiated, which would support the more important role of host adaptation (selection) than gene flow in shaping population genetic structure. Previous findings revealing that $E$. lata isolates from different hosts were all infectious in grapevine (46) do not allow testing for a higher performance on the host of origin, which would suggest host specialization. Accordingly, we conducted cross-inoculations of the two most economically and historically important hosts of E. lata, grapevine and apricot, to test for host specialization. Evidence for host-based genetic differentiation and/or host specialization would help focus management strategies on the most significant source of inoculum.

\section{MATERIALS AND METHODS}

Experimental design. From October 2009 to June 2011, hierarchical sampling was carried out in three locations of California (Napa, San Benito, and Solano Counties). In each location, three host plants, grapevine (Vitis vinifera), apricot (Prunus armeniacae), and willow (Salix sp.), were sampled, resulting in nine sampling sites (Table 1). Willow was considered the most important source of inoculum among native hosts by Trouillas and Gubler (46), based on the relatively high incidence of perithecia (sexual fruiting bodies) observed in nature. A total of 182 E. lata isolates were collected. Spatial distances among the three locations ranged from 60 to $350 \mathrm{~km}$ (Fig. 1). Within each location, spatial distances among the vineyard, apricot orchard, and riparian area did not exceed $6 \mathrm{~km}$. This sampling scheme was designed to determine whether isolates from one host, across locations, were genetically more similar than isolates from different hosts in the same location.

At each location, vineyards, apricot orchards, and riparian areas were surveyed for plants with general dieback symptoms that are typical of all trunk diseases of grape (i.e., dead spur positions, shoot dieback, poor growth) and for the more specific, characteristic foliar symptoms of Eutypa dieback (in grapevine: stunting of shoots, shortening of internodes, chlorosis, necrosis and cupping of leaves; in apricot: intense gummosis, branch death, and wilting of leaves) (8). At each sampling site, between 47 and 118 plants were sampled for fungal isolation (Table 1). Monosporous isolates were recovered from perithecia and mass-hyphal isolates from necrotic wood, as previously described (46). Masshyphal isolates were further purified through hyphal-tip subcultures. Disease incidence was estimated as the proportion of plants from which E. lata was successfully isolated. To test equality of disease incidence between host plants, confidence intervals for incidence differences (CI) were calculated as

$$
\mathrm{CI}=p_{a}-p_{b} \pm 1.96 \times \sqrt{ }\left[\left(p_{a} \times q_{a} /\left(n_{a}-1\right)\right)+\left(p_{b} \times q_{b} /\left(n_{b}-1\right)\right)\right]
$$

where $p_{a}$ and $p_{b}$ are disease incidences in each host; $q_{a}$ and $q_{b}=$ $1-p ; n_{a}$ and $n_{b}$ are the number of samples from each host; and 1.96 corresponds to the $95 \%$ confidence level.

Population genetic analyses. All 182 isolates were genotyped with nine polymorphic microsatellite markers that have been suitable for $E$. lata population studies $(6,45)$, using published polymerase chain reaction (PCR) conditions (45). Briefly, cultures were incubated on potato dextrose agar ([PDA], Difco, Detroit, MI) at $25^{\circ} \mathrm{C}$ in dark for 14 days. DNA was extracted from aerial mycelia (DNeasy Plant kit, Qiagen). Species identity was confirmed by PCR amplification and sequencing of the rDNA internal transcribed spacer region (ITS) (ITS1 and ITS4 [47]). Based on phylogenetic analyses of ITS sequences, these 182 isolates clustered with type specimens in maximum likelihood analyses and were identified as E. lata (data not shown). Allele assignments were performed on an ABI PRISM 3130xl sequencer (GENEMARKER v.1.75, SoftGenetics LLC). All 182 isolates were genotyped twice, to ensure repeatability. The allelic data set contained no missing data.

The number of repeated multilocus haplotypes, within and across sampling sites, was determined using GENALEX 6 (33), and this number was used to estimate, at each sampling site, the

TABLE 1. Hosts of origin, geographic locations, and genetic diversity of Eutypa lata populations from three host plants in California (182 total isolates)

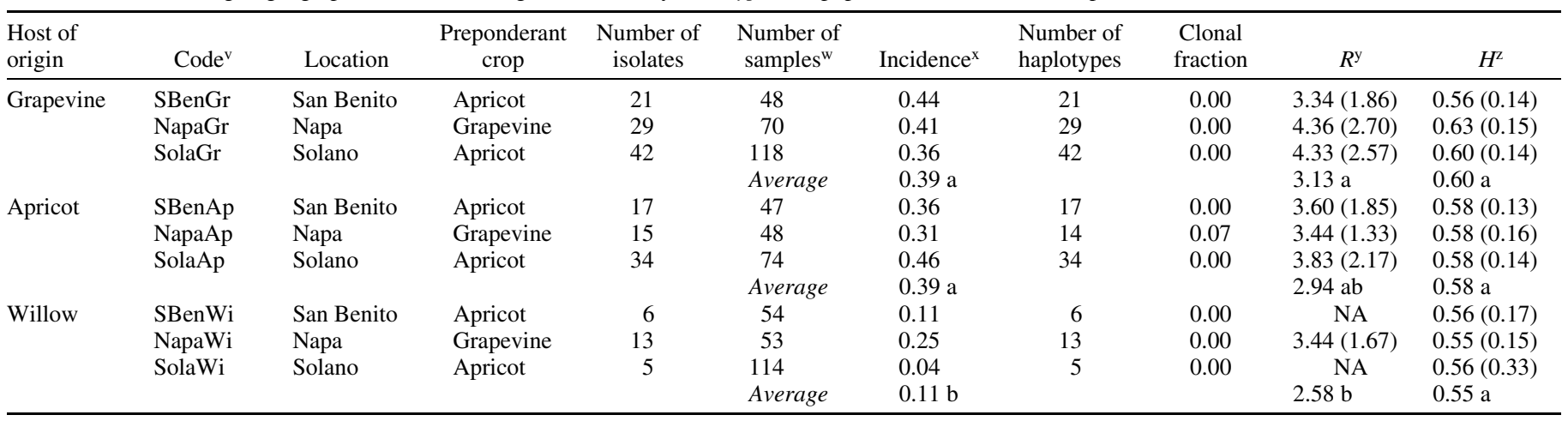

${ }^{\mathrm{v}}$ Population code for each host at each location.

${ }^{w}$ Number of plants from which a symptomatic wood sample was collected for fungal isolations.

${ }^{x}$ Proportion of plants from which $E$. lata was cultured. Values followed by different letters are significantly different, based on lack of overlap of $95 \%$ confidence intervals.

${ }^{y}$ Mean allelic richness (standard deviation in parentheses) per host plant, averaged across loci and sampling sites (37). Values followed by different letters are significantly different (assessed after 5,000 random permutations of haplotypes across hosts; $P>0.05$ ). NA $=$ not applicable; Number of haplotypes $<10$.

${ }^{\mathrm{z}}$ Mean gene diversity (standard deviation in parentheses) per host plant, averaged across loci and sampling sites (32). Values followed by different letters are significantly different (assessed after 5,000 random permutations of haplotypes across hosts; $P>0.05$ ). 
clonal fraction as 1 - ([number of different haplotypes]/[total number of isolates]). Genetic diversity was estimated within each sampling site by estimating the allelic richness $(R)$ and unbiased gene diversity $(H)$ (32). Isolates from willow in San Benito and in Solano were excluded from these analyses due to small sample sizes ( $<10$ haplotypes). Allelic richness $R$ corresponds to the mean number of alleles per locus. Gene diversity $H$ corresponds to the average probability across loci to draw, at random, different alleles in the same sampling site. $R$ and $H$ were adjusted for variable sample size, based on rarefaction estimates (37), using FSTAT 2.9.3.2 (23). The statistical significance of differences in $H$ and $R$ among host plants, across locations, was assessed in twosided permutation tests at 5,000 permutations.

Hierarchical analyses of molecular variance (AMOVA) were used to quantify the partitioning of molecular variation at different hierarchical levels (12). Two distinct analyses were performed: samples were grouped either according to their host of origin or their location (i.e., county) of origin. The AMOVA framework incorporates haplotype divergence into a matrix of squared-distances among all pairs of haplotypes used to produce estimates of variance components and $F$-statistic analogs, designated as $\Phi$-statistics (12). GENALEX was used to compute and test the statistical significance of $\Phi$-statistics based on 999 random permutations of haplotypes.

To examine population subdivision, we used a Bayesian method of assignment implemented in the program STRUCTURE 2.3.1 (39). This program uses a Markov Chain Monte Carlo algorithm to assign multilocus haplotypes to a genetic cluster, assuming Hardy-Weinberg equilibrium and minimizing linkage disequilibrium among loci within clusters. Analyses were performed both using uniform priors and using the location of origin or host of origin of isolates as prior information to assist clustering (model LocPrior [24]). The likelihood of the posterior probability distributions was computed for each number of clusters $(K)$ from 1 to 12 . To check for consistency of likelihood values for each $K$ value between runs, each $K$ was simulated six times, with a run length of 300,000 iterations after the specified burn-in (300,000 iterations), using the admixture model of genetic ancestry and the correlated model of allele frequency (14). We estimated the number of clusters as deltaK, which is based on the second-order rate of change of the likelihood of posterior probability of the data $L(K)$ between successive $K$ values (11).

Cross-pathogenicity tests. A total of 24 isolates was selected for cross-inoculations, with 12 isolates from San Benito and 12 isolates from Napa, in February 2012. From each location, six isolates originated from grapevine and six from apricot. Symptom development of Eutypa dieback in grapevine woody tissue requires an incubation period $\geq 12$ months. As such, this was a 14-month experiment encompassing two greenhouses, in which we propagated the perennial hosts from dormant plant material. Inoculum consisted of a 3-mm-diameter agar plug colonized by actively growing mycelium (PDA, Difco Laboratories). The 24 isolates were inoculated to grapevine ('Thompson Seedless') and apricot ('Patterson'), and noninoculated controls were mockinoculated with sterile PDA to give a total of 50 isolate $\times$ host combinations. Two replicate experiments were conducted 2 weeks apart in two separate greenhouses $(n=$ six plants per treatment, 600 total plants). In each greenhouse, plants were arranged in a completely randomized design.

Grapevines were propagated from 1-year-old dormant cuttings of 'Thompson Seedless.' Cuttings were cut to uniform length $(\approx 30 \mathrm{~cm})$ containing three nodes, surface-sterilized in $1 \%$ sodium hypochlorite (Clorox, Oakland, CA) for $15 \mathrm{~min}$, rinsed in water overnight, and then callused in a mixture of perlite and vermiculite $(1: 1, \mathrm{vol} / \mathrm{vol})$, at $30^{\circ} \mathrm{C}$ and $100 \% \mathrm{RH}$ for 3 weeks. Once root and shoot initials emerged from the callus tissue, the stem of each cutting was wounded at approximately $3 \mathrm{~cm}$ below the uppermost node with a 3-mm-cork borer, and a 3-mm mycelial plug from a 10-day-old PDA culture was inserted into the wound and sealed with Vaseline and Parafilm. Noninoculated controls were "mockinoculated" with a sterile PDA plug. After inoculation, cuttings were submerged in melted paraffin wax (Gulf Wax, Royal Oak Enterprises, Atlanta, GA) within $4 \mathrm{~cm}$ of the roots and potted in UC mix (5) amended with slow-release fertilizer (Osmocote Pro 24-4-9, Scotts, Marysville, OH).

Apricots were propagated from dormant, bare root trees of 'Patterson' $(\approx 1 \mathrm{~cm}$ diameter) grafted onto Nemaguard rootstock. On the day of inoculations, apricot trees were taken out of cold storage $\left(2^{\circ} \mathrm{C}\right)$, and the inoculation and potting procedures were identical to those for the grapevines, except with no waxing step. Grapevines and apricots were grown in the greenhouse at the University of California Experimental Station in Davis from February 2012 to April 2013 (natural sunlight photoperiod, $25 \pm$ $1^{\circ} \mathrm{C}$ [day], $18 \pm 3^{\circ} \mathrm{C}$ [night]). In early November, plants were pruned and the temperature was decreased $\left(10 \pm 2{ }^{\circ} \mathrm{C}\right.$ [day], $4 \pm$ $2{ }^{\circ} \mathrm{C}$ [night]) until the end of January. Plants were watered twice per week for $15 \mathrm{~min}$ using a drip-irrigation system $\left(0.51 \mathrm{~h}^{-1}\right)$.

The performance of $E$. lata isolates was based on two distinct measures: the incidence of foliar symptoms and the extent of wood lesions in inoculated stems. Following bud break in the second growing season (mid-February 2013), plants were monitored for foliar symptoms twice per week for 6 weeks. Incidence of foliar symptoms was calculated as the proportion of plants per treatment expressing the following characteristic symptoms of Eutypa dieback: necrotic areas on some leaves, dwarfed leaves with necrosis, reduction in leaf and shoot size, rapid dieback of stunted shoots, and/or no shoot growth. Characteristic foliar symptoms on apricot included a rapid dieback of the shoots above the inoculation site during the growing season or no shoot growth in the second growth cycle. Wood symptoms were evaluated 14 months after inoculation (April 2013). Plants were removed from the soil; roots and shoots were excised; and bark was scraped from the woody stem. Stems were surface-sterilized in $1 \%$ sodium hypochlorite solution for $2 \mathrm{~min}$, rinsed with tap water,

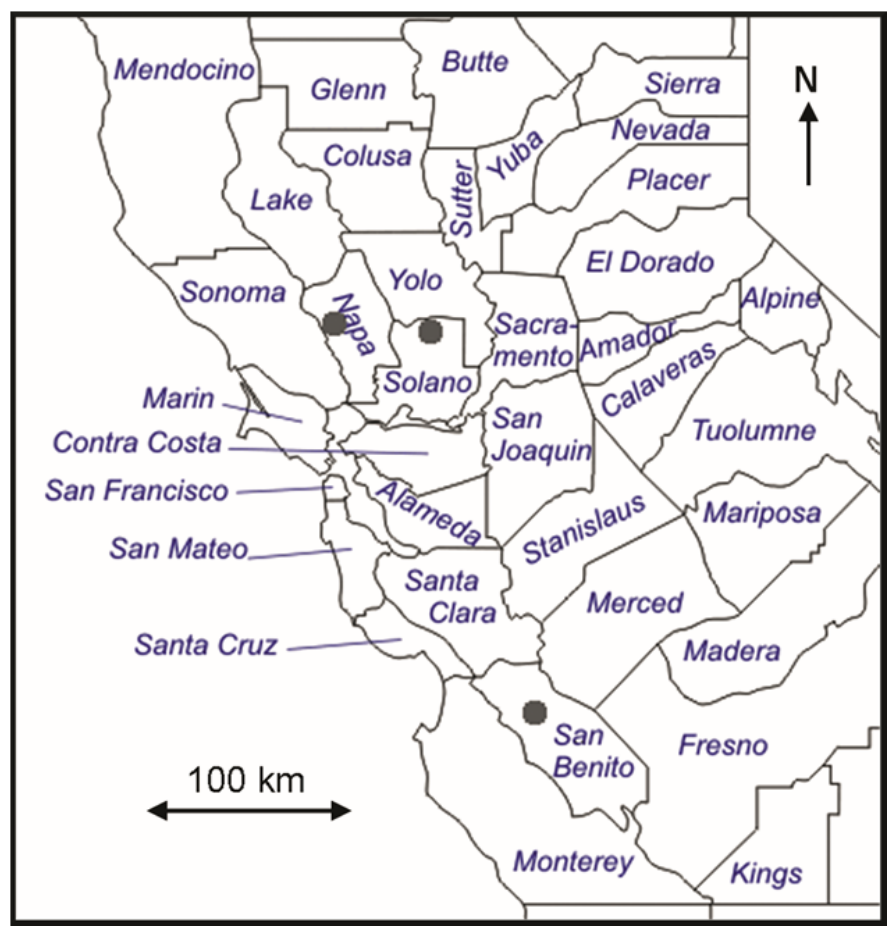

Fig. 1. Locations in northern California (Napa, San Benito, and Solano counties) where Eutypa lata populations were examined. Within each location, a vineyard and apricot orchard, both with symptoms of Eutypa dieback, were sampled for the pathogen. Willows were also sampled within each location, in adjacent riparian areas. 
and cut longitudinally. The length of wood lesions extending above and below the point of inoculation was measured with a caliper.

There are two ways to test for host specialization in a "common garden" experiment, as conducted here: (i) by comparing the performance of a pathogen population from a given host in different hosts (the "home versus away" criterion), or (ii) by comparing the performance of a pathogen population in its native habitat (i.e., host of origin) with that of another pathogen population from a different habitat (the "local vs. foreign" criterion) (26). In both cases, the relevant statistic is the significance of the interaction between the pathogen population (from the host of origin) and test host (host inoculated). The incidence of foliar symptoms (pathogenicity) was compared among treatments using a generalized linear model with a binomial error distribution and a logit link function (GLIMMIX procedure of SAS version 9.2; SAS Institute, Cary, NC). When testing for a host specialization pattern (higher average incidence of foliar symptoms on host of sampling), the host of origin, test host, and their two-way interactions were considered fixed effects. In the model, replicate plants and fungal isolates were considered random effects; they were nested within test host and host of origin, respectively. Contrasts allowed testing the "home vs. away" criterion (comparisons between the two hosts for one pathogen population) and the "local vs. foreign" criterion (comparisons between the two pathogen populations on one host). Severity of wood symptoms (i.e., wood lesion length; aggressiveness) was compared among treatments using an analysis of variance (ANOVA; MIXED procedure of SAS); host specialization was tested as previously described for incidence of foliar symptoms.

\section{RESULTS}

Disease incidence. General dieback symptoms were observed in all three hosts and in all three locations. Characteristic Eutypa dieback symptoms (intense gummosis, branch death, and wilting of leaves) were observed in some apricot trees at each location. Similarly, diagnostic symptoms were observed in several grapevines at locations surveyed during spring months. Perithecia of the fungus, which were embedded in a black stroma located within the bark, were observed on each host species. Based on recovery of the fungus in culture, the incidence of $E$. lata in vineyards and apricot orchards ranged from 31 to $46 \%$ (Table 1). In contrast, incidence in willow trees was lower, ranging from 4 to $25 \%$. Across locations, the average incidence in grapevine and apricot was $39 \%$ and did not differ significantly (95\% CI of -0.10 to $0.10, P=0.05,401 \mathrm{df}$ ). Disease incidence averaged across locations was significantly lower in willow (11\%) than in grapevine (95\% CI of 0.21 to $0.35, P=0.05,453 \mathrm{df}$ ). Similarly, disease incidence was significantly lower in willow than in apricot (95\% CI of 0.20 to $0.36, P=0.05,386 \mathrm{df}$ ).

Genetic diversity. Only two repeated multilocus haplotypes were identified from the same sampling site (apricot in Napa,
NapaAp). The clonal fraction was null in all but one sampling site (NapaAp, Table 1). In addition, two pairs of repeated haplotypes were identified from distinct sampling sites: two identical haplotypes were identified from apricot in Napa and Solano, and two identical haplotypes were identified from apricot in Solano and San Benito. Gene diversity $(H)$ was homogenous across sampling sites and varied between 0.55 and 0.63. Differences in allelic richness $(R)$ were revealed across sampling sites: the mean number of alleles per locus, estimated for 13 haplotypes (the smallest sample size in the data set), ranged from 3.34 (SBenGr) to 4.36 (NapaGr). When $H$ and $R$, averaged across sampling sites per host, were compared among hosts (grapevine, apricot, willow), significant differences in allelic richness were revealed $(P=$ 0.048). Consistently for these two measures of genetic diversity, sampling sites from grapevine were the most genetically diverse (Table 1).

The hierarchical AMOVA, first using location as a grouping factor, revealed that $99 \%$ of the genetic variation was found within sampling sites, and differences among locations did not contribute to total genetic variation (Table 2). Genetic differences among sampling sites within a location contributed to $1 \%$ of genetic variance. When host was used as a grouping factor, results were similar and $99 \%$ of the genetic variation was found within sampling sites. The results of the AMOVA analyses, therefore, indicated that there were no significant differences in pathogen populations among sampling sites from different locations or from different hosts. The low genetic differentiation among samples from different hosts was illustrated by the negative and nonsignificant estimator of genetic differentiation $\left(\Phi_{\mathrm{RT}}=-0.004\right.$; $P=0.67$; Table 2), suggesting high levels of gene flow among the three hosts.

Clustering analysis. The Bayesian method of assignment of multilocus haplotypes detected population subdivision, as evidenced by the estimated modal values of the statistic delta $K$ (Fig. 2). Analyses using sampling location as prior information suggested the presence of three genetic clusters, whereas analyses using host of sampling as prior information did not reveal any clear population structure (Fig. 2). Analyses that made no use of prior population information revealed that either three or five genetic clusters of multilocus haplotypes best described the genetic variation. According to these results, we presented membership coefficients to each cluster for $K=3$ and $K=5$ (Fig. 3). Bar plots for $K=3$, using either no prior population information or sampling location as prior information to assist clustering, revealed a high proportion of multilocus haplotypes with admixed ancestry $(<50 \%$ probability of membership to any cluster). In contrast, bar plots representing the membership coefficients to each cluster for $K=5$ (no use of prior population information) revealed a clearer pattern of population subdivision, while the proportion of multilocus haplotypes with admixed ancestry was low (16 out of 182 haplotypes). Assessment of membership probabilities relative to the host of origin of each haplotype did

TABLE 2. Hierarchical analysis of molecular variance (AMOVA), partitioning the genetic variation of Eutypa lata haplotypes grouped by location (San Benito, Napa, and Solano) or by host (grapevine, apricot, and willow) ${ }^{\mathrm{x}}$

\begin{tabular}{|c|c|c|c|c|c|}
\hline Source of variation & $\mathrm{df}$ & $\begin{array}{l}\text { Variance } \\
\text { components }\end{array}$ & $\%$ variation & $\Phi$-statistics ${ }^{\mathrm{y}}$ & $P$ values $^{\mathrm{z}}$ \\
\hline Among locations & 2 & 0.00 & 0 & $\Phi_{\mathrm{RT}}=-0.004$ & 0.61 \\
\hline Among sampling sites within locations & 6 & 3.75 & 1 & $\Phi_{\mathrm{PR}}=0.006$ & 0.34 \\
\hline Among hosts & 2 & 0.00 & 0 & $\Phi_{\mathrm{RT}}=-0.004$ & 0.67 \\
\hline Among sampling sites within hosts & 6 & 3.76 & 1 & $\Phi_{\mathrm{PR}}=0.006$ & 0.35 \\
\hline Within sampling sites & 173 & 674.53 & 99 & $\Phi_{\mathrm{PT}}=0.001$ & 0.46 \\
\hline
\end{tabular}

${ }^{x}$ Sampling site is the location $\times$ host combination (e.g., grapevines in San Benito or SBenGr).

${ }^{\text {y }} \Phi$-statistics are analogues of $F$-statistics, estimated through AMOVA (12). $\Phi_{\mathrm{RT}}=$ proportion of variance among regions, relative to total variance. $\Phi_{\mathrm{PR}}=$ proportion of variance among populations within regions, relative to variance among and within populations. $\Phi_{\mathrm{PT}}=$ proportion of variance among populations, relative to total variance.

${ }^{z}$ Probability that a statistic obtained from 999 random permutations of haplotypes across the data set is greater than the value observed. 
not reveal any genetic clustering by host plant. Indeed, most genetic clusters consisted of haplotypes from all hosts and locations. For example, cluster 4 included haplotypes from each of the nine sampling sites (Fig. 4). The five genetic clusters were not supported by any reasonable biological interpretation, such as geography or host of origin. The lack of substantive biological reasons to support the outputs of these analyses led us to consider this identified genetic structure as marginal.

Cross-pathogenicity tests. Inoculations conducted in the greenhouse on grapevine and apricot plants revealed a large variation in both the severity of wood symptoms and the incidence of foliar symptoms among the 24 isolates (Fig. 5). When inoculated to grapevines, there was a smaller range in length of wood lesions (55 to $145 \mathrm{~mm}$ ) than on apricot (50 to $360 \mathrm{~mm}$ ). Ranges of foliar symptom incidence were similar in both hosts (approximately 20 to $100 \%)$. Some isolates caused large wood lesions and frequent foliar symptoms on grapevine and apricot (e.g., SBen204, SBen218). By contrast, some isolates caused small wood lesions and a low incidence of foliar symptoms on both hosts (e.g., SBen015). In a few cases, some isolates seemed better adapted to one host than the other; for example, isolate SBen020 caused larger lesions and a higher incidence of foliar symptoms on apricot than on grapevine (Fig. 5).

Host specialization. A significant difference in E. lata pathogenicity was found between isolates from the two hosts of origin, with higher average incidence of foliar symptoms for isolates from apricot than isolates from grapevine $(P=0.037$; Table 3$)$. Contrasts revealed greater incidence of foliar symptoms of grapevine isolates when inoculated to grapevine than to apricot ("home versus away" comparison, $P=0.024$; Table 3, Fig. 6A), suggesting a better performance on the host of origin. Nonetheless, this pattern of host specialization was not observed for apricot isolates; they caused greater incidence of foliar symptoms on grapevine than on apricot. The absence of a significant host of origin $\times$ test host interaction $(P=0.072$; Table 3$)$ and the absence of crossing reaction norms (Fig. 6A) revealed no clear hostspecialization pattern for $E$. lata based on incidence of foliar symptoms.

Host of origin, test host, and their interaction had significant effects on the length of wood lesions, indicating a pattern of host specialization based on this life-history trait (Table 4). Indeed, isolates from apricot caused larger wood lesions than isolates from grapevine when inoculated to apricot ("local vs. foreign" comparison, $P=0.0006$; Table 4, Fig. 6B). The two significant "home vs. away" comparisons revealed an overall higher severity of wood lesions of isolates from both hosts when inoculated to apricot (Table 4 and Fig. 6B). For example, grapevine isolates caused larger wood lesions in apricot than in grapevine $(P=$ 0.0002; Table 4). These results indicated an overall greater level of aggressiveness in (or susceptibility of) apricot, independently of the host in which these isolates originated.

\section{DISCUSSION}

We tested the hypothesis of host specialization in the woodcanker pathogen E. lata, and our results did not provide evidence for host-associated genetic differentiation or preferential host exploitation in this pathogen. The pathogen population infecting multiple hosts in California was genetically homogenous, based on our finding of no genetic structuring according to either host or geographic origins. The assessment of host specialization to grapevine and apricot for two life-history traits, the pathogen's ability to cause foliar symptoms and to cause large wood lesions, did not support a consistent pattern of host adaptation. These results support an overall greater role of gene flow than selection in structuring the pathogen population in California. The three host plants examined may not exert strong divergent selection pressures on the pathogen population, and free migration among hosts may be facilitated by the large population sizes and high fecundity that characterize fungal pathogens.

As concerns about "pathogen pollution" and emerging infectious diseases of plants increases (4), a thorough understanding of population dynamics of pathogens that infect multiple host species is required (10). The effect of host species diversity on disease risk in a host of interest can have several outcomes depending on the relative properties of each host (27). Different hosts usually have different "reservoir potential," corresponding to the product of the proportion of plants infected by the pathogen and the probability that this host will serve as a source of inoculum (29). With respect to $E$. lata, the fungus produces sexual fruiting bodies on any infected and dying hosts (46). Hence, once infected, grapevine, apricot, and willow constitute a source of infectious propagules. As a first approximation, we can thus consider that the reservoir potential of each host depends only on the pathogen incidence (i.e., prevalence) in each host. Our findings illustrate the contrasted reservoir potential of the three host plants examined; grapevine and apricot had similar incidences of the pathogen $(39 \%)$, whereas willow had a significantly lower reservoir potential, with an incidence of $11 \%$, averaged across the three locations. These results support the hypothesis that cultivated crops, such as grapevine and apricot, are more important sources of inoculum than are wild plants, such as willow, present in riparian areas $(13,38)$.

Our finding of higher genetic diversity of E. lata populations on grapevine and apricot further support this hypothesis. When measured in terms of allelic richness, the genetic diversity of the $E$. lata populations on willow was significantly lower than those of populations on grapevine or apricot. This lower genetic diversity of populations on willow is in agreement with a smaller effective population size of E. lata populations in riparian areas. As previously discussed (45), E. lata is a wound pathogen, and grapevine and apricot are regularly and severely "wounded" when they are pruned each winter, which is a cultural practice used to maintain high crop productivity. In contrast, willow is a wild host; it is not pruned and thus has fewer infection courts. This may

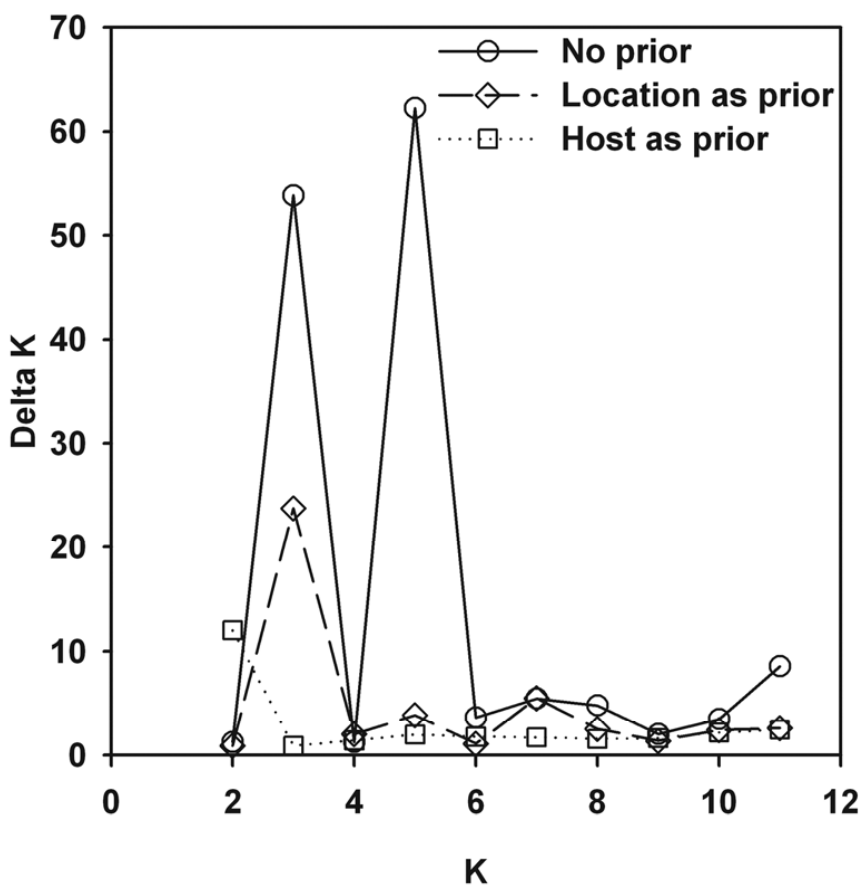

Fig. 2. Change in the statistic delta $K$ relative to the number of clusters $K$ ( 1 to 12) tested on the 182 Eutypa lata multilocus haplotypes with the program STRUCTURE. Estimates of delta $K$ are based on the second order rate of change of the likelihood of posterior probability of the data between successive $K$ values. 
explain the low genetic diversity of E. lata in willow, relative to the higher incidence, effective population size, and genetic diversity observed in E. lata populations in apricot orchards and vineyards.

In populations of plant-pathogenic fungi, the spatial scale of pathogen dispersal usually affects the spatial scale of genetic differentiation, which in turn may affect the scale of local adaptation (i.e., higher performance on sympatric than on allopatric genotypes of a given host [44]). In the present study, we anticipated that a pattern of adaptation of E. lata to a particular host may not be detectable at a local spatial scale (i.e., between an adjacent vineyard and orchard) because of high dispersal levels among hosts that may "swamp" locally adapted genotypes. Instead we hypothesized that such a pattern may be more likely detectable at a regional spatial scale (i.e., San Benito versus Napa), because these two locations are separated by $360 \mathrm{~km}$, which may restrict habitat connectivity. However, our finding of no significant genetic differentiation of E. lata populations suggests that gene flow among hosts and locations at both local and regional scales prevents genetic differentiation in this pathogen. High levels of gene flow were previously shown among $E$. lata populations from different continents $(36,45)$. Gene flow at local spatial scales is likely a result of passive ascospore dispersal by wind, whereas gene flow at continental scales is more likely a result of human-mediated transport of infected plant material (45). A high level of gene flow among local E. lata populations in California may promote the mixing of locally adapted genotypes and migrant, maladapted genotypes in each local pathogen population.

As for the homogenizing effect of gene flow that hinders the differentiating effect of selection, sexual recombination also tends to break up locally adapted allelic combinations (linkage dis-

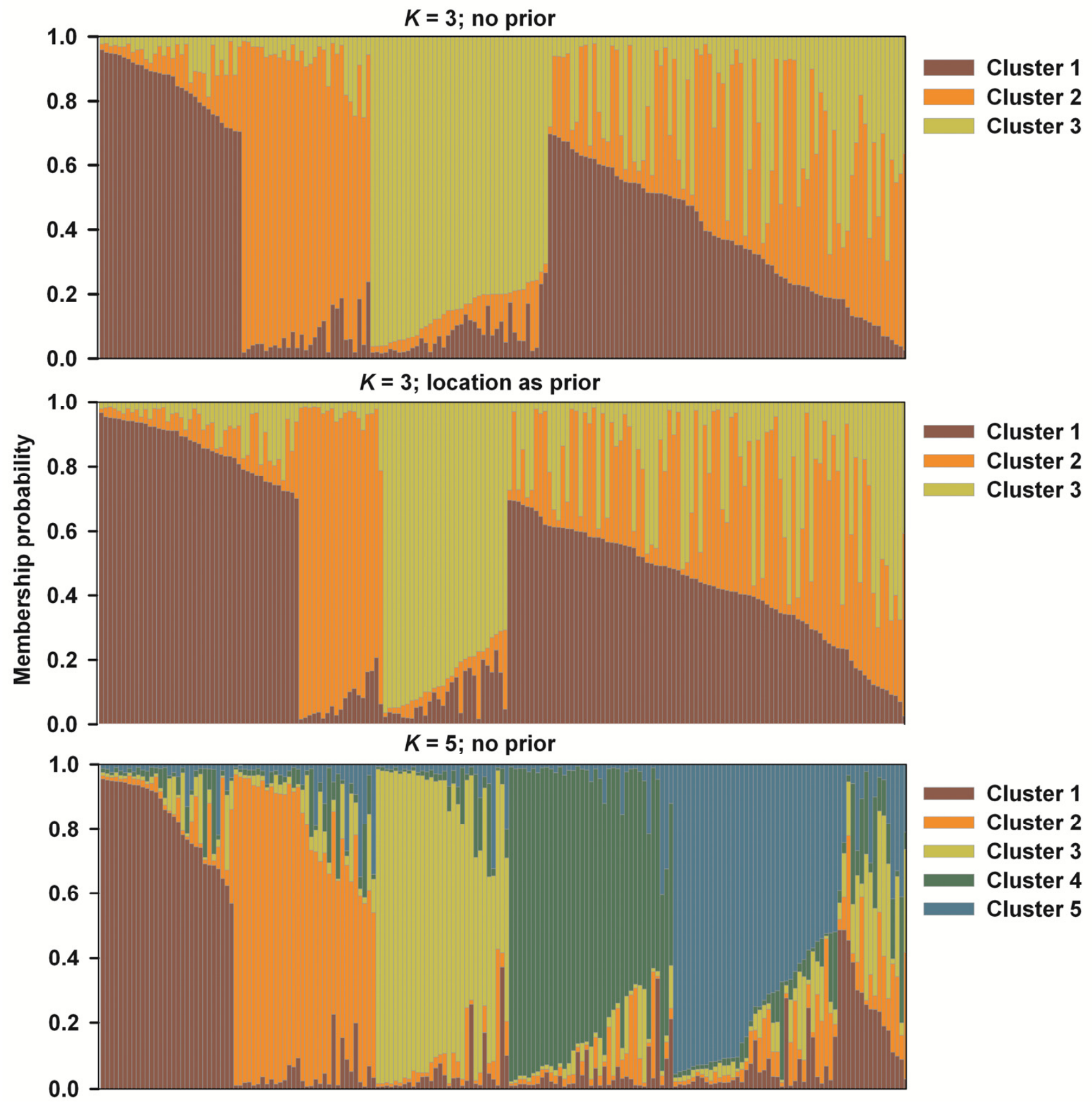

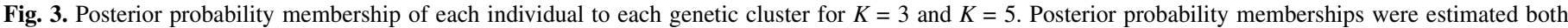

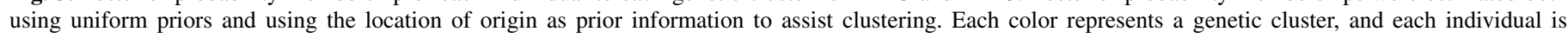
represented by a vertical line partitioned into colored segments whose lengths represent the admixture proportions from $K$ clusters. 
equilibrium created by selection), except when genes controlling habitat specialization and mate choice (i.e., assortative mating) are identical or physically linked (19). Although we did not test for multilocus or pairwise linkage disequilibrium in the present study, previous genetic analyses of E. lata populations with the same set of microsatellite markers (45) and different markers $(35,36)$ support the hypothesis that E. lata is a random mating species. Indeed, repeated haplotypes are rare. It has been suggested that life history traits of many plant-pathogenic fungi, especially when mating takes place within the host plant, favor the fixation of alleles controlling host specialization (21). Host adaptation induces pleiotropically assortative mating, which can eventually lead to reproductive isolation and ecological speciation (20). Hence, if California is a recent geographic area occupied by this pathogen (45), a short evolutionary timescale may have prevented host adaptation; host range expansion may be too recent to generate divergences within the pathogen population. Also, we cannot reject the hypothesis that our neutral genetic markers are not suited to detect differentiation among host-adapted popu-

TABLE 3. Results of the generalized linear mixed model assessing host specialization (effects of host of origin [apricot and grapevine], test host, and their interaction) on foliar symptom incidence caused by Eutypa lata

\begin{tabular}{llrl}
\hline Source of variation & df & $F$ value & $P>F$ \\
\hline Main effects & & & \\
Host of origin & 1 & 13.41 & 0.0370 \\
Test host & 1 & 0.17 & 0.6907 \\
Host of origin $\times$ test host & 1 & 3.97 & 0.0719 \\
Contrasts & & & \\
Local vs. foreign (grapevine) & 1 & 1.18 & 0.3015 \\
Local vs. foreign (apricot) & 1 & 3.08 & 0.1069 \\
Home vs. away (grapevine) & 1 & 15.27 & 0.0240 \\
Home vs. away (apricot) & 1 & 1.46 & 0.2520 \\
\hline
\end{tabular}

${ }^{z}$ The "local vs. foreign" test compares foliar symptom incidence caused by isolates from both hosts of origin on the test host (in parentheses). The "home vs. away" test compares foliar symptom incidence caused by isolates from the host of origin (in parentheses) on both test hosts. lations; instead, examining genes under positive selection may allow the detection of host specialization (3). Alternatively, if host adaptation evolved in source populations of the pathogen before introduction to California, patterns of host adaptation should still be detectable because mating occurs within host plants, thereby facilitating the fixation of alleles controlling host adaptation in local pathogen populations (21). Our results suggest that such a pattern of host adaptation was absent in source populations of this pathogen.

The characterization of 24 E. lata isolates for foliar symptom incidence and wood lesion length provided further insights into the phenotypic diversity in populations of this fungus. The population examined in California can be considered polymorphic, based on the variation in these two life-history traits. Such polymorphism can be considered adaptive when natural selection promotes phenotypic diversity (31), as is the case when heterogenous environments (e.g., distinct hosts) favor frequency-dependent selection (40). However, our findings do not support such adaptive polymorphism. For instance, the absence of a pattern of

TABLE 4. Results of the analysis of variance assessing host specialization (effects of host of origin [apricot and grapevine], test host, and their interaction) on the length of wood lesions caused by Eutypa lata

\begin{tabular}{lcrr}
\hline Source of variation ${ }^{\mathrm{z}}$ & df & $F$ value & $P>F$ \\
\hline Main effects & & & \\
Host of origin & 1 & 4.17 & 0.0417 \\
Test host & 1 & 68.15 & $<0.0001$ \\
Host of origin $\times$ test host & 1 & 8.66 & 0.0034 \\
Contrasts & 1 & 0.42 & 0.5149 \\
Local vs. foreign (grapevine) & 1 & 11.88 & 0.0006 \\
Local vs. foreign (apricot) & 1 & 13.78 & 0.0002 \\
Home vs. away (grapevine) & 1 & 64.12 & $<0.0001$ \\
Home vs. away (apricot) & &
\end{tabular}

z The "local vs. foreign" test compares length of wood lesions caused by isolates from both hosts of origin on the test host (in parentheses). The "home vs. away" test compares length of wood lesions caused by isolates from the host of origin (in parentheses) on both test hosts.

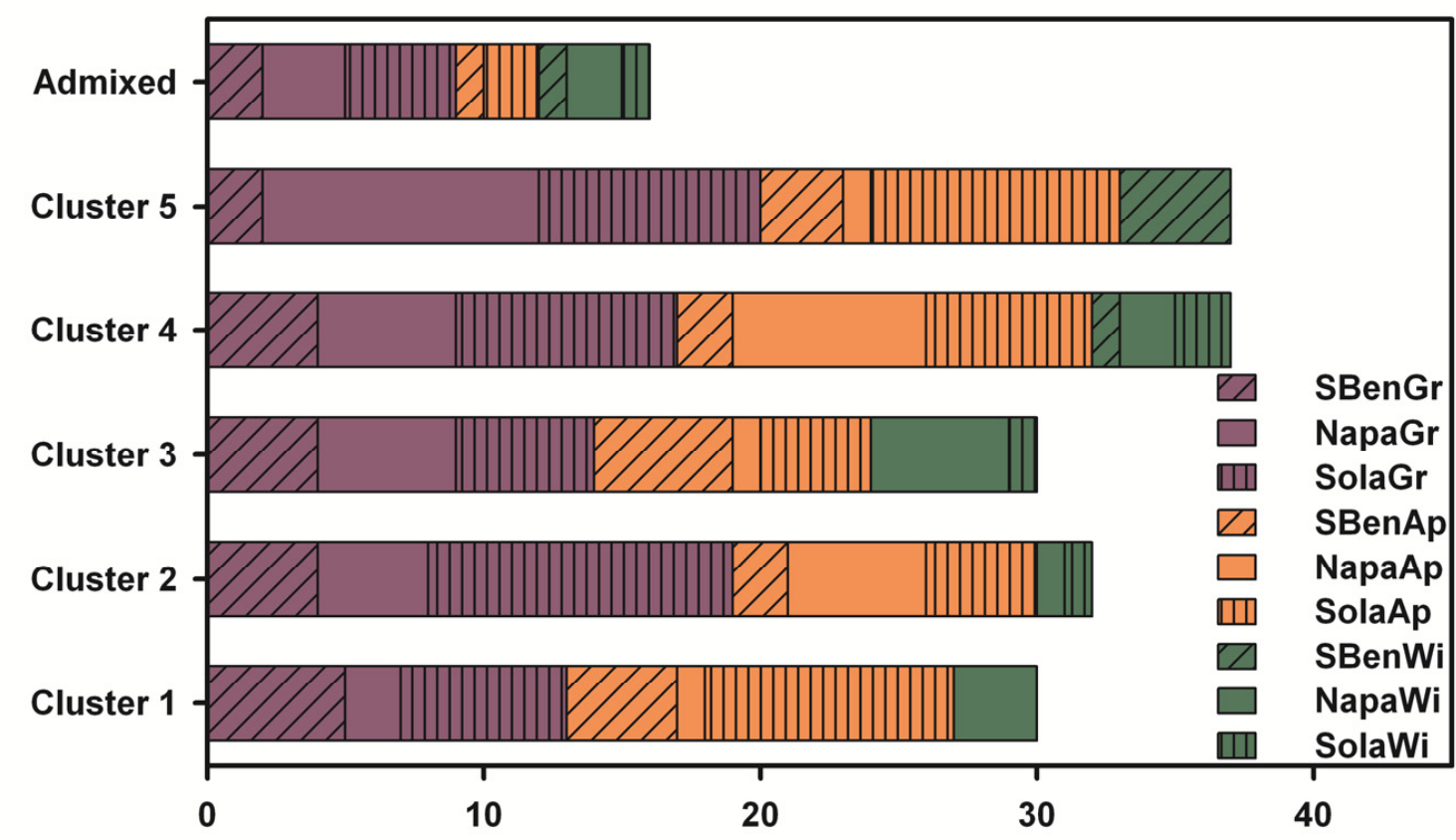

\section{Number of isolates}

Fig. 4. Distribution of Eutypa lata isolates among the five genetic clusters, identified with the program STRUCTURE. Each colored pattern within bars corresponds to the sampling location of origin, as detailed in Table 1. SBen $=$ San Benito, Napa = Napa, Sola $=$ Solano, Gr $=$ grapevine, Ap $=$ apricot, $\mathrm{W}=$ willow. 
host specialization for the incidence of foliar symptoms does not suggest that the host plants exert a strong selection pressure for this trait. Moreover, some isolates caused severe foliar symptoms on both hosts, regardless of their host of origin. Considering the large genotypic polymorphism detected among the isolates studied and the apparent lack of adaptive phenotypic diversity, such genetic variation could be considered adaptive if it allows the pathogen to perform efficiently in an increased range of niches, relative to less genetically diverse pathogen populations; this hypothesis remains to be tested. The large phenotypic variation observed among the 24 isolates for severity of wood symptoms also precluded the detection of a pattern of host speciali- zation. Some isolates could be considered adapted to grapevine and maladapted to apricot (e.g., SBen022); other isolates could be considered adapted to apricot but not to grapevine (e.g., Napa215); and isolates could be maladapted to both hosts (e.g., SBen015) or adapted to both hosts (e.g., SBen207). Overall, our finding of such diversified phenotypes, representing variable performance levels of isolates in different hosts, suggests a generalist strategy in E. lata that may enable this species to persist and reproduce on a large range of hosts.

The broad host range of E. lata in California (46) and our findings supporting a generalist strategy in this wood-decay fungus suggest that "preadapted" genotypes were present in the

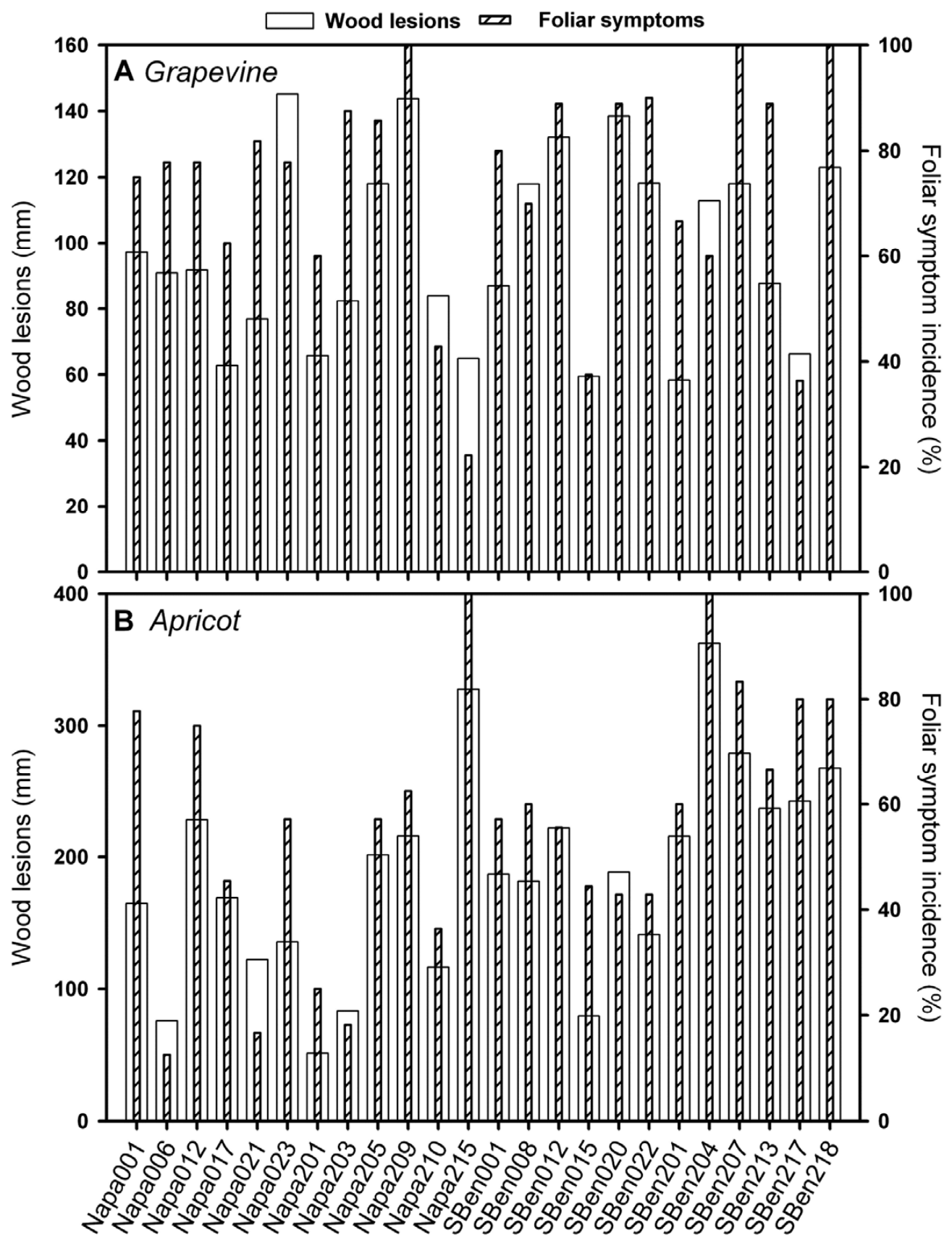

Isolate

Fig. 5. Length of wood lesions and incidence of foliar symptoms caused by 24 E. lata isolates on $\mathbf{A}$, grapevine, and $\mathbf{B}$, apricot plants. Isolates from Napa and San Benito are labeled Napa and SBen, respectively. Isolates recovered from grapevine are labeled from 001 to 023 , whereas isolates recovered from apricot are labeled 201 to 218. Incidence of foliar symptoms was assessed at the beginning of the second growth cycle, at 12 months after inoculation in the greenhouse. Severity of wood symptoms was expressed as wood lesion length $(\mathrm{mm})$ and was measured in the inoculated stems at 14 months after inoculation. 
pathogen population at the time of introduction to California, and such genotypes were able to use novel resources for survival and reproduction (i.e., phenotypic plasticity). Organisms have a range of environments in which they can survive and reproduce, and this range is larger than the sole environments in which they have evolved (2). This corresponds to the process of ecological fitting, implying that the pathogen traits necessary to infect a plant (i.e., for the plant to serve as a host) did not result from a long coevolutionary history with that host, but rather that such traits evolved under different environmental conditions $(2,25)$. We hypothesize that the introduced population of E. lata in California evolved as a soft-rot pathogen in its native range, which allowed subsequent host-range expansion in novel areas where new hosts with similar physiology and defense responses were present (i.e., host resource tracking). Assuming that defense responses among these related hosts were conserved, the pathogen may have been able to infect hosts it had not previously encountered (1). In conclusion, E. lata and possibly other wood-decay fungi infecting exposed wood may possess the characteristics that would allow them to infect new hosts they have not coevolved with in the past through ecological fitting. Future studies on adaptive patterns in wood-decay fungi might consider ecological fitting as a cause of host-range expansion.

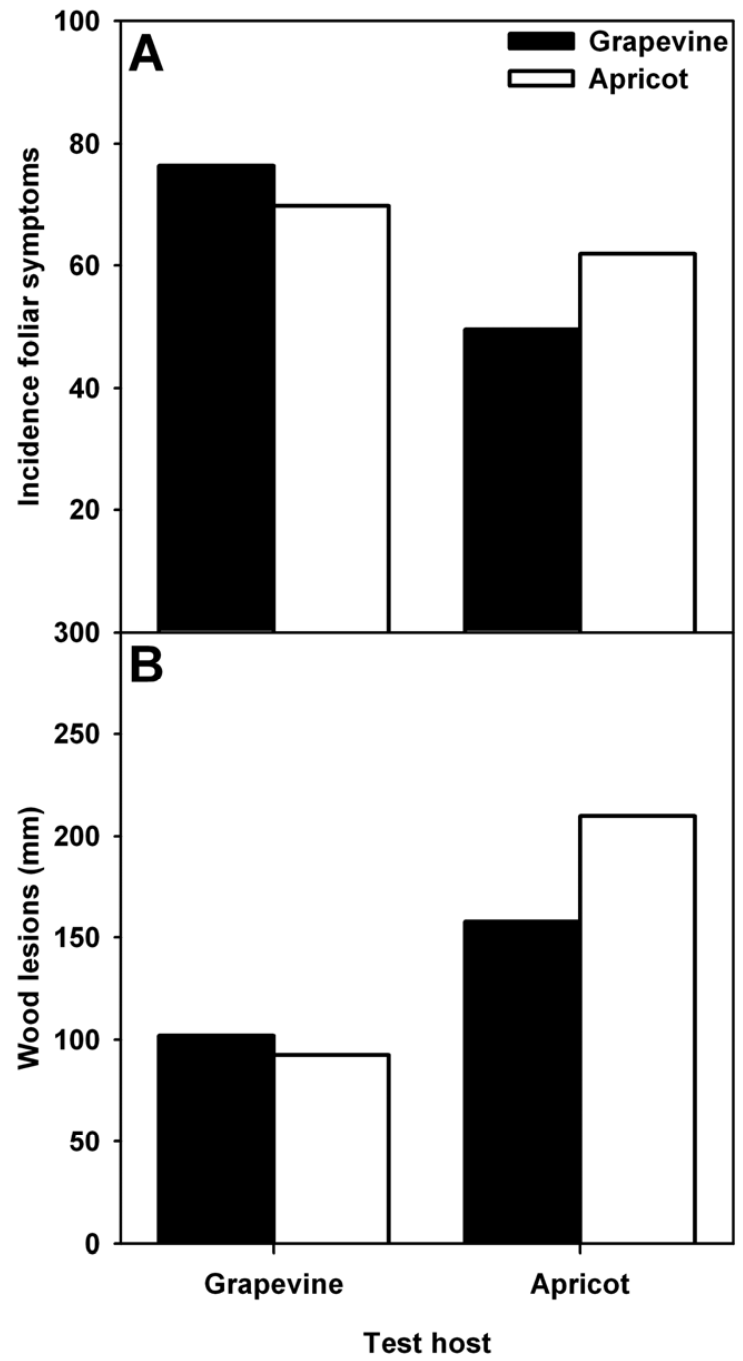

Fig. 6. Host specialization in Eutypa lata. A, Mean incidence of foliar symptoms, and $\mathbf{B}$, severity of wood symptoms by $E$. lata isolates from grapevine and apricot on grapevine and apricot plants. $P$ values from contrast tests are indicated in Tables 3 and 4 for foliar symptom incidence and severity of wood lesions, respectively.

\section{ACKNOWLEDGMENTS}

Research was funded by grant number 2011-962 to K. Baumgartner from the American Vineyard Foundation. Reviews of this manuscript were provided by J. Montarry (INRA, Le Rheu, France) and P. Gladieux (University of California, Berkeley, CA). USDA is an equal opportunity provider and employer. Authors declare no conflict of interest. Mention of trade names or commercial products is solely for the purpose of providing specific information and does not imply recommendation or endorsement by the U.S. Department of Agriculture. USDA is an equal opportunity provider and employer.

\section{LITERATURE CITED}

1. Agosta, S. J. 2006. On ecological fitting, plant-insect associations, herbivore host shifts, and host plant selection. Oikos 114:556-565.

2. Agosta, S. J., and Klemens, J. A. 2008. Ecological fitting by phenotypically flexible genotypes: Implications for species associations, community assembly and evolution. Ecol. Lett. 11:1123-1134.

3. Aguileta, G., Refregier, G., Yockteng, R., Fournier, E., and Giraud, T. 2009. Rapidly evolving genes in pathogens: Methods for detecting positive selection and examples among fungi, bacteria, viruses and protists. Discussion. Infect. Genet. Evol. 9:656-670.

4. Anderson, P. K., Cunningham, A. A., Patel, N. G., Morales, F. J., Epstein, P. R., and Daszak, P. 2004. Emerging infectious diseases of plants: Pathogen pollution, climate change and agrotechnology drivers. Trends Ecol. Evol. 19:535-544.

5. Baker, K. F. 1957. UC system for producing healthy container-grown plants. University of California Press, Berkeley.

6. Baumgartner, K., Bergemann, S. E., Fujiyoshi, P., Rolshausen, P. E., and Gubler, W. D. 2009. Microsatellite markers for the grapevine pathogen, Eutypa lata. Mol. Ecol. Res. 9:222-224.

7. Brunner, J. L., LoGiudice, K., and Ostfeld, R. S. 2008. Estimating reservoir competence of Borrelia burgdorferi hosts: Prevalence and infectivity, sensitivity, and specificity. J. Med. Entomol. 45:139-147.

8. Carter, M. V. 1991. The status of Eutypa lata as a pathogen. International Mycological Institute, Phytopathological Paper 32. CAB International, Wallingford, England.

9. Carter, M. V., Bolay, A., English, H., and Rumbos, I. 1985. Variation in the pathogenicity of Eutypa lata (=Eutypa armeniacae). Aust. J. Bot. 33:361-366.

10. Dobson, A. 2004. Population dynamics of pathogens with multiple host species. Am. Nat. 164:S64-S78.

11. Evanno, G., Regnaut, S., and Goudet, J. 2005. Detecting the number of clusters of individuals using the software STRUCTURE: A simulation study. Mol. Ecol. 14:2611-2620.

12. Excoffier, L., Smouse, P. E., and Quattro, J. M. 1992. Analysis of molecular variance inferred from metric distances among DNA haplotypes: Application to human mitochondrial DNA restriction data. Genetics 131:479-491.

13. Fabiszewski, A. M., Umbanhowar, J., and Mitchell, C. E. 2010. Modeling landscape-scale pathogen spillover between domesticated and wild hosts: Asian soybean rust and kudzu. Ecol. Appl. 20:582-592.

14. Falush, D., Stephens, M., and Pritchard, J. K. 2003. Inference of population structure using multilocus genotype data: Linked loci and correlated allele frequencies. Genetics 164:1567-1587.

15. Farr, D., and Rossman, A. 2014. Fungal Databases, Systematic Mycology and Microbiology Laboratory, ARS, USDA. http://nt.ars-grin.gov/ fungaldatabases/

16. Fournier, E., and Giraud, T. 2008. Sympatric genetic differentiation of a generalist pathogenic fungus, Botrytis cinerea, on two different host plants, grapevine and bramble. J. Evol. Biol. 21:122-132.

17. Gandon, S. 2004. Evolution of multihost parasites. Evolution 58:455-469.

18. Gandon, S., and Michalakis, Y. 2002. Local adaptation, evolutionary potential and host-parasite coevolution: Interactions between migration, mutation, population size and generation time. J. Evol. Biol. 15:451-462.

19. Gavrilets, S. 2004. Fitness landscapes and the origin of species. Princeton University Press, NJ.

20. Giraud, T., Gladieux, P., and Gavrilets, S. 2010. Linking the emergence of fungal plant diseases with ecological speciation. Trends Ecol. Evol. 25:387-395.

21. Giraud, T., Villareal, L. M., Austerlitz, F., Le Gac, M., and Lavigne, C. 2006. Importance of the life cycle in sympatric host race formation and speciation of pathogens. Phytopathology 96:280-287.

22. Gladieux, P., Guerin, F., Giraud, T., Caffier, V., Lemaire, C., Parisi, L., Didelot, F., and Le Cam, B. 2011. Emergence of novel fungal pathogens by ecological speciation: Importance of the reduced viability of immigrants. Mol. Ecol. 20:4521-4532.

23. Goudet, J. 1995. FSTAT (Version 1.2): A computer program to calculate 
F-statistics. J. Hered. 86:485-486.

24. Hubisz, M. J., Falush, D., Stephens, M., and Pritchard, J. K. 2009. Inferring weak population structure with the assistance of sample group information. Mol. Ecol. Res. 9:1322-1332.

25. Janzen, D. H. 1985. On ecological fitting. Oikos 45:308-310.

26. Kawecki, T. J., and Ebert, D. 2004. Conceptual issues in local adaptation. Ecol. Lett. 7:1225-1241.

27. Keesing, F., Holt, R. D., and Ostfeld, R. S. 2006. Effects of species diversity on disease risk. Ecol. Lett. 9:485-498.

28. Lardner, R., Mahoney, N., Zanker, T. P., Molyneux, R. J., and Scott, E. S. 2006. Secondary metabolite production by the fungal pathogen Eutypa lata: Analysis of extracts from grapevine cultures and detection of those metabolites in planta. Aust. J. Grape Wine Res. 12:107-114.

29. LoGiudice, K., Duerr, S. T., Newhouse, M. J., Schmidt, K. A., Killilea, M. E., and Ostfeld, R. S. 2008. Impact of host community composition on Lyme disease risk. Ecology 89:2841-2849.

30. Mahoney, N., Lardner, R., Molyneux, R. J., Scott, E. S., Smith, L. R., and Schoch, T. K. 2003. Phenolic and heterocyclic metabolite profiles of the grapevine pathogen Eutypa lata. Phytochemistry 64:475-484.

31. Moran, N. A. 1992. The evolutionary maintenance of alternative phenotypes. Am. Nat. 139:971-989.

32. Nei, M. 1978. Estimation of average heterozygosity and genetic distance from a small number of individuals. Genetics 89:583-590.

33. Peakall, R., and Smouse, P. E. 2006. GENALEX 6: Genetic analysis in Excel. Population genetic software for teaching and research. Mol. Ecol. Notes 6:288-295.

34. Peever, T. L. 2007. Role of host specificity in the speciation of Ascochyta pathogens of cool season food legumes. Eur. J. Plant Pathol. 119:119-126.

35. Péros, J. P., and Berger, G. 1999. Diversity within natural progenies of the grapevine dieback fungus Eutypa lata. Curr. Genet. 36:301-309.

36. Péros, J. P., and Berger, G. 2003. Genetic structure and variation in aggressiveness in European and Australian populations of the grapevine dieback fungus, Eutypa lata. Eur. J. Plant Pathol. 109:909-919.

37. Petit, R. J., El Mousadik, A., and Pons, O. 1998. Identifying populations for conservation on the basis of genetic markers. Conserv. Biol. 12:844855 .

38. Power, A. G., and Mitchell, C. E. 2004. Pathogen spillover in disease epidemics. Am. Nat. 164:S79-S89.

39. Pritchard, J. K., Stephens, M., and Donnelly, P. 2000. Inference of population structure using multilocus genotype data. Genetics 155:945959.

40. Ravigné, V., Dieckmann, U., and Olivieri, I. 2009. Live where you thrive: Joint evolution of habitat choice and local adaptation facilitates specialization and promotes diversity. Am. Nat. 174:E141-E169.

41. Rolshausen, P. E., Greve, L. C., Labavitch, J. M., Mahoney, N. E., Molyneux, R. J., and Gubler, W. D. 2008. Pathogenesis of Eutypa lata in grapevine: Identification of virulence factors and biochemical characterization of cordon dieback. Phytopathology 98:222-229.

42. Rouxel, M., Mestre, P., Comont, G., Lehman, B. L., Schilder, A., and Delmotte, F. 2012. Phylogenetic and experimental evidence for hostspecialized cryptic species in a biotrophic oomycete. New Phytol. 197:251-263.

43. Stukenbrock, E. H., and McDonald, B. A. 2008. The origins of plant pathogens in agro-ecosystems. Annu. Rev. Phytopathol. 46:75-100.

44. Thrall, P. H., Burdon, J. J., and Bever, J. D. 2002. Local adaptation in the Linum marginale-Melampsora lini host-pathogen interaction. Evolution 56:1340-1351.

45. Travadon, R., Baumgartner, K., Rolshausen, P. E., Gubler, W. D., Sosnowski, M. R., Lecomte, P., Halleen, F., and Péros, J. P. 2012. Genetic structure of the fungal grapevine pathogen Eutypa lata from four continents. Plant Pathol. 61:85-95.

46. Trouillas, F. P., and Gubler, W. D. 2010. Host range, biological variation, and phylogenetic diversity of Eutypa lata in California. Phytopathology 100:1048-1056.

47. White, T. J., Bruns, T., Lee, S., and Taylor, J. 1990. Amplification and direct sequencing of fungal ribosomal RNA genes for phylogenetics. In: PCR Protocols: A Guide to Methods and Applications. M. A. Innis, eds. Academic Press, New York. 